\title{
Longitudinal growth of infants born to HIV-1-infected mothers in Belo Horizonte, Brazil
}

\author{
Maria Arlene Fausto ${ }^{1, *}$, Mariângela Carneiro ${ }^{2}$, Carlos Maurício F Antunes ${ }^{2}$, \\ Enrico Antônio Colosimo ${ }^{3}$ and Jorge A Pinto ${ }^{4}$ \\ 'Departamento de Alimentos, Escola de Nutrição, Universidade Federal de Ouro Preto, Ouro Preto, Minas \\ Gerais 35400-000, Brazil: ${ }^{2}$ Departamento de Parasitologia, Laboratório de Epidemiologia, Instituto de Ciências \\ Biológicas, Universidade Federal de Minas Gerais, Belo Horizonte, Minas Gerais, Brazil: ${ }^{3}$ Departamento de \\ Estatística, Instituto de Ciências Exatas, Universidade Federal de Minas Gerais, Belo Horizonte, Minas Gerais, \\ Brazil: ${ }^{4}$ Departamento de Pediatria, Faculdade de Medicina, Universidade Federal de Minas Gerais, \\ Belo Horizonte, Minas Gerais, Brazil
}

\section{Submitted 24 May 2007: Accepted 16 April 2008: First published online 27 May 2008}

\begin{abstract}
Objective: To prospectively evaluate growth parameters assessed by weight and length in infected and uninfected infants born to HIV-1-infected mothers and followed from birth to 18 months.

Methods: A cohort consisting of ninety-seven uninfected and forty-two infected infants born to HIV-infected mothers enrolled from 1995 to 2004, and admitted during their first 3 months of life at a referral Pediatric AIDS Clinic in Belo Horizonte, Brazil. Infants were followed until 18 months of age. Data were analysed using mixed-effects linear regression models for weight and length fitted by restricted maximum likelihood.

Results: Infected infants contributed to 466 weight and 411 recumbent length measurements. Uninfected infants provided 924 weight and 907 length measurements. Mean birth weight and length were similar in both groups, $3 \cdot 1(\mathrm{sD} 0 \cdot 4)$ and $3 \cdot 0(\mathrm{SD} 0 \cdot 5) \mathrm{kg}$, and $48 \cdot 7(\mathrm{SD} 1.4)$ and $48 \cdot 8(\mathrm{SD} 2 \cdot 9) \mathrm{cm}$ for uninfected and infected infants, respectively. However, HIV-1 infection had an early impact in growth impairment: at 6 months of age, HIV-infected children were $1 \mathrm{~kg}$ lighter and $2 \mathrm{~cm}$ shorter than the uninfected.

Conclusions: Growth faltering in weight, but not length, in HIV-infected children in Brazil is more marked than that reported in a European cohort, probably reflecting background nutritional deficiencies and concomitant infections. In these settings, early and aggressive nutritional management in HIV-1infected infants should be a priority intervention associated with the antiretroviral therapy.
\end{abstract}

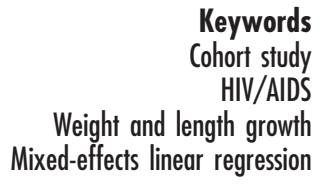

Keywords

Chort study

IV/AIDS Mixed-effects linear regression
The HIV epidemic began in Brazil in the 1980s and by the end of 2003 UNAIDS estimated that 660000 Brazilians had already been infected with the virus ${ }^{(1)}$, corresponding to $40.5 \%$ of the total HIV-infected population of Latin America. Between 1980 and 2005 the number of AIDS cases reported to the Brazilian Ministry of Health was $371827^{(2)}$, of which $9965(2 \cdot 7 \%)$ were perinatally infected children. Antiretroviral therapy (ART) for the prevention of mother-to-child transmission of HIV-1 (PMTCT) was introduced in Brazil in 1995, resulting in significant reduction of transmission rates ${ }^{(2)}$.

The assessment of weight at birth and of child growth is one of the indicators of their nutritional status. In developed countries, HIV vertically infected children present similar weight and length at birth compared to those of non-infected children ${ }^{(3)}$. Differences in weight and length growth of infected and uninfected children start to appear in the first months of life ${ }^{(3,4)}$.

The growth assessment of HIV-infected children is important both to determine the disease stage and prognosis and to assess the effectiveness and toxicity of ART regimens and to study the nutritional implications of the infection $^{(5)}$. Growth seems to be one of the most sensitive indicators of the disease progression in children living with $\mathrm{HIV}_{\text {AIDS }}^{(6)}$. Even in children who have been using antiretroviral drugs, the absence of growth is a poor prognostic indicator. Weight gain is also an important indicator of ART effectiveness $^{(7)}$. For these reasons, anthropometric methods are useful to monitor the disease progression and to assess response to treatment. 
Studies involving infants born to HIV-1-infected mothers can provide useful insights into the nutritional implications of the infection, and can contribute to the establishment of intervention strategies for this segment of the population. The objective of the present study was to prospectively evaluate growth parameters assessed by weight and recumbent length in infected and uninfected infants born to HIV-1-infected mothers and followed from birth to 18 months.

\section{Patients and methods}

\section{Study design and population}

This cohort study was conducted at the Universidade Federal de Minas Gerais (UFMG) Pediatric HIV Clinic, in Belo Horizonte, Brazil. Infants born to HIV-1-infected mothers from 1995 to 2003 and admitted at the clinic within the first 3 months of life were eligible to participate. The subjects were followed up to 18 months of age. Clinical and laboratory data were collected using standardised data forms, coded and entered in a database. The study was approved by the UFMG Ethical Committee.

Determination of HIV infection was based on the Brazilian National AIDS Program (NAP) algorithm $^{(8)}$ : (i) the existence of two samples, taken after 2 weeks of life, testing positive for HIV according to a DNA-PCR (Amplicor ${ }^{\circledR}$; Roche, Basel, Switzerland) or an RNA-PCR (NASBA $^{\circledR}$; Organon-Teknica, Boxtel, The Netherlands) method; (ii) the persistence of HIV antibodies as detected by ELISA and Western blot after 18 months of age; or (iii) the development at any age of a clinical condition compatible with AIDS according to the Centers for Disease Control and Prevention (CDC)/94 classification system for infants under 13 years ${ }^{(9)}$. The criteria to establish the absence of HIV infection were: (i) two negative HIV DNA-PCR or RNA-PCR samples, taken at ages 2 weeks to 4 months and 4-6 months; and (ii) two negative anti-HIV (ELISA) serum tests performed after 6 months of age, with a minimum interval between tests of 2 months, and the absence of clinical conditions compatible with AIDS.

\section{Antbropometric measurements}

Weight in kilograms and length in centimeters of ninetyseven HIV-uninfected and forty-two HIV-infected infants were measured during regular visits by trained paediatricians. The measurements were carried out according to standard procedures ${ }^{(10)}$. An electronic paediatric scale (Indústria Filizola S/A, São Paulo, Brazil), weighing capacity from $125 \mathrm{~g}$ to $15 \mathrm{~kg}$, accuracy of $5 \mathrm{~g}$, was used to weigh children and body length was measured using a horizontal wooden stadiometer with the infant in recumbent position. Infected and uninfected infants contributed with 466 weight and 411 length measurements and 924 weight and 907 length measurements, respectively.
The median follow-up times were $15 \cdot 2$ months (range 6.8-18.0 months) and $14 \cdot 3$ months (range 6.3-18.6 months) for the HIV-infected and uninfected infants, respectively.

\section{Statistical analysis}

Statistical analyses were performed using S-Plus $2000^{(11)}$ and Stata $6 \cdot 0^{(12)}$ software, and in all cases the level of significance was established at $5 \%$.

The mean values of weights and lengths of the two groups, taken at the time of birth, were compared using Student's $t$ test $^{(13)}$. The distribution of the categorical variables according to the two clinical groups was evaluated using the $\chi^{2}$ and the Fisher exact tests ${ }^{(13)}$. HIV-infected children were classified according to the 1994 CDC classification for children under the age of 13 years ${ }^{(9)}$.

The mixed-effects linear regression models for weight and stature were fitted using the restricted maximum likelihood method. The LME function provided by the S-Plus 2000 software $^{(14)}$ was used in order to find the estimates. Comparison of the models was based on the Akaike Information Criteria and on the maximum likelihood ratio test. The effect of extra random terms was evaluated using the restricted maximum likelihood ratio test.

The approach described by Diggle et al. ${ }^{(15)}$ was used to evaluate the covariance structure of the anthropometric models. The covariates included in the statistical analysis were exposure to prophylactic ART regimens ( $1=$ yes, $0=$ no), gender of child $(1=$ male, $0=$ female $)$, clinical group $(1=$ HIV-infected, $0=$ uninfected $)$, gestational period $(0=$ more than 36 weeks, $1=$ less than 36 weeks $)$ and polynomial age (months ${ }^{n} ; n=1, n=2$ ).

Based on the values of weight and length, the growth rates of the different groups of children were obtained from the final equations produced by the mixed-effects models. The average growth rates were stratified according to age groups and compared with those obtained from the European Collaborative Study ${ }^{(3)}$, using the large-sample $z$ test.

\section{Results}

Premature births, defined as children born before 36 weeks of gestation, were observed only among the HIVinfected group (22\%). There were no significant differences with respect to birth weight and length between HIV-uninfected and infected children born at term. The mean birth weight and length of uninfected children were $3 \cdot 1(\mathrm{SD} 0 \cdot 4) \mathrm{kg}$ and $48.7(\mathrm{SD} 1 \cdot 4) \mathrm{cm}$. Similarly, infected children had a mean birth weight of $3 \cdot 0($ SD 0.5$) \mathrm{kg}$ and a mean length of $48 \cdot 8(\mathrm{sD} 2 \cdot 9) \mathrm{cm}$. Males comprised $54.6 \%$ of the uninfected and $52.4 \%$ of the infected population. Exposure to ART for PMTCT was reported by 91/95 $(95 \cdot 8 \%)$ of non-transmitting mothers and by $28 / 42$ (66.7\%) of transmitting mothers $(P<0 \cdot 0001)$. 
During the follow-up period, ART was initiated in 31 out of the $42(73 \cdot 8 \%)$ infected children, at a mean age of $8 \cdot 7$ months (range $0 \cdot 8-17 \cdot 1$ ). Dual nucleoside analogue reverse transcriptase inhibitors (NRTI) were the initial regimen in eleven $(35.5 \%)$ subjects. Highly active antiretroviral therapy (HAART) was used as the initial regimen by the remaining twenty (64.5\%) subjects. Eighteen subjects were on a protease inhibitor (PI)-containing HAART regimen and two on a non-PI HAART regimen (2 NRTI +1 non-nucleoside analogue reverse transcriptase inhibitor). There were no changes on the initial ART regimen during the observation period.

The median age at first visit was 0.9 months (range $0 \cdot 2-3.5$ ) and 1.7 months (range $0 \cdot 1-3.5$ ) for uninfected and infected children, respectively. At admission, most infected children were asymptomatic (27/42 or $64 \cdot 3 \%)$. At 1 year of age, $33 \cdot 3 \%$ of the evaluable infected children presented with category C disease (12/36). During the follow-up period, mortality rate among the infected children was $7 \cdot 1 \%(95 \%$ CI $1 \cdot 5,19 \cdot 5)$. No deaths were observed in the uninfected children.

As recommended by the Brazilian $\mathrm{NAP}^{(8)}$, artificial formula feeding was provided free of charge to all HIV1-exposed infants for the first 6 months of age, regardless of their infection status.

Table 1 shows the final models for weight and recumbent length. The variable group was included in the model in order to detect if there were differences between HIVinfected and uninfected children with respect to weight. The results show that as length increased, boys became heavier than girls, while the interaction between length and age indicates that the effects of length on the weight of the infants increased with age. Moreover, HIV-infected children were lighter than the corresponding uninfected children, and this difference in body weight increased until the end of the first year of life. At 6 months of age, the average weight difference between uninfected and infected children was approximately $1 \mathrm{~kg}$, while at 12 months the difference was $1.3 \mathrm{~kg}$. The growth rate of infants, expressed in terms of body weight, decreased as a function of increasing age (Fig. 1), particularly within the group of uninfected children: after 12 months of age, the growth rate within the HIV-infected group was actually higher than that of the uninfected group.

The variable group was included in the recumbent length model for the same purpose as in the weight model. The model presented in Table 1 establishes that the differences in length between boys and girls increased with age: thus, at 6 months boys were $2.0 \mathrm{~cm}$ taller than girls, while at 12 months the average difference was $2 \cdot 8 \mathrm{~cm}$. The interaction between group and age polynomial demonstrates that HIV-infected children were smaller than uninfected children, on average $2.8 \mathrm{~cm}$ shorter at 6 months. The difference in length between HIV-infected and uninfected infants increased up to 8 months of age, after which it began to diminish, although HIV-infected children were always smaller than uninfected children. For both groups, the linear growth rate decreased as a function of increasing age (Fig. 2); the growth rate of HIV-infected children was lower than that

Table 1 Weight and length models for HIV-infected and uninfected infants of a cohort of Belo Horizonte (Minas Gerais, Brazil), born between 1995 and 2004

\begin{tabular}{|c|c|c|c|c|c|c|c|c|}
\hline \multirow[b]{2}{*}{ Variables } & \multicolumn{4}{|c|}{ Weight values } & \multicolumn{4}{|c|}{ Length values } \\
\hline & $\beta$ & SD & $95 \% \mathrm{Cl}$ & $P$ & $\beta$ & SD & $95 \% \mathrm{Cl}$ & $P$ \\
\hline Intercept & $-2 \cdot 46$ & $0 \cdot 39$ & $-3 \cdot 23,-1 \cdot 69$ & $<0.0001$ & 48.94 & 0.33 & $48 \cdot 29,49 \cdot 60$ & $<0.0001$ \\
\hline Sex & $-1 \cdot 01$ & $0 \cdot 32$ & $-1 \cdot 65,-0 \cdot 37$ & 0.002 & 0.51 & $0 \cdot 41$ & $-0 \cdot 31,1 \cdot 33$ & 0.22 \\
\hline Clinical group & $-0 \cdot 14$ & 0.07 & $-0.27,-0.0009$ & 0.048 & -0.73 & 0.46 & $-1 \cdot 64,0 \cdot 18$ & $0 \cdot 12$ \\
\hline Stature & $0 \cdot 11$ & 0.008 & $0 \cdot 09,0 \cdot 12$ & $<0.0001$ & & & & \\
\hline Age (month) & 1.03 & 0.06 & $0 \cdot 91,1 \cdot 16$ & $<0.0001$ & 3.02 & 0.09 & $2 \cdot 83,3 \cdot 20$ & $<0.0001$ \\
\hline $\mathrm{Age}^{2}\left(\right.$ month $\left.^{2}\right)$ & -0.04 & 0.006 & $-0.05,-0.03$ & $<0.0001$ & -0.08 & 0.006 & $-0 \cdot 10,-0.07$ & $<0.0001$ \\
\hline \multicolumn{9}{|l|}{ Interactions } \\
\hline Clinical group $\times$ age & $-0 \cdot 19$ & 0.03 & $-0.25,-0.13$ & $<0.0001$ & -0.52 & $0 \cdot 13$ & $-0.77,-0.27$ & $<0.0001$ \\
\hline Clinical group $\times$ age $^{2}$ & 0.008 & 0.002 & $0.005,0.01$ & $<0.0001$ & 0.03 & 0.007 & $0.01,0.04$ & 0.0003 \\
\hline Sex $\times$ age & & & & & $0 \cdot 31$ & $0 \cdot 12$ & $0.09,0.54$ & 0.006 \\
\hline Sex $\times$ age $^{2}$ & & & & & -0.01 & 0.007 & $-0.03,-0.0008$ & 0.04 \\
\hline Sex $\times$ stature & 0.02 & 0.006 & $0.01,0.04$ & 0.0002 & & & & \\
\hline Length $\times$ age & -0.008 & 0.0009 & $-0.01,-0.006$ & $<0.0001$ & & & & \\
\hline Length $\times$ age $^{2}$ & 0.0004 & 0.00006 & $0.0003,0.0005$ & $<0.0001$ & & & & \\
\hline \multicolumn{9}{|l|}{ Random effects } \\
\hline Intercept & & $0 \cdot 27$ & & & & $2 \cdot 14$ & & \\
\hline Age & & $0 \cdot 14$ & & & & 0.56 & & \\
\hline $\mathrm{Age}^{2}$ & & 0.006 & & & & 0.03 & & \\
\hline Residual & & $0 \cdot 31$ & & & & $1 \cdot 54$ & & \\
\hline Measurements $(M)$ & & $1 \cdot 313$ & & & & $1 \cdot 318$ & & \\
\hline Number of children $(n)$ & & 139 & & & & 139 & & \\
\hline
\end{tabular}

$\beta=$ coefficient estimates. 


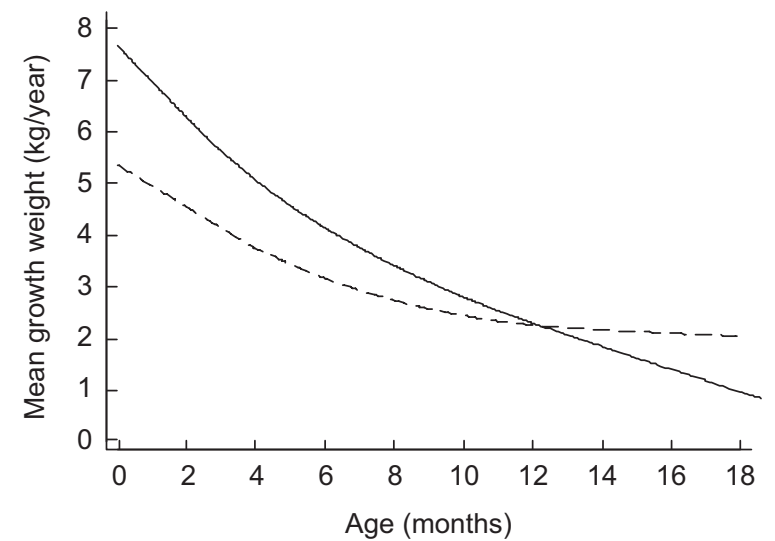

Fig. 1 Growth rate, expressed in terms of change in weight (kg/year), of the uninfected (-) and HIV-infected (- - -) groups within a cohort of infants born between 1995 and 2004 in Belo Horizonte, Minas Gerais, Brazil

of uninfected children until 8 months of age and subsequently higher.

Table 2 shows the rates of growth of HIV-infected and uninfected children in this cohort estimated from the weight and recumbent length models. The information was compared with the data presented in the European study ${ }^{(3)}$. Within the age range of 1-24 months, both HIV-infected and uninfected Brazilian children presented a growth rate by weight smaller than in the European study. Within the age range of 1-11 months, the growth rate by length of uninfected children was higher than that observed in the European study ${ }^{(3)}$, while within the age range of 12-24 months the growth rate was smaller. The growth rate by length of HIV-infected children was similar in both cohorts within the age range of $1-5$ months. Within the age range of 6-24 months, the HIV-infected children in our cohort presented a growth rate by length higher than that of the European cohort. However, the comparison between the two cohorts is limited due to the fact that data in our cohort were collected only from 0 to 18 months of age.

\section{Discussion}

The results of the present study demonstrate that differences in weight and length between uninfected and HIVinfected infants emerge at an early age and increase as the children get older. The growth of HIV-infected children is clearly affected by the disease, as shown by the differences in weight ( $1 \mathrm{~kg}$ lighter) and length ( $2 \mathrm{~cm}$ smaller) of this group compared with uninfected children at 6 months of age.

The catch-up on weight and length seen among the infected children at the end of first year of life is probably related to the response to ART. As reported by others, control of viral replication is associated with sustained growth $^{(16,17)}$.

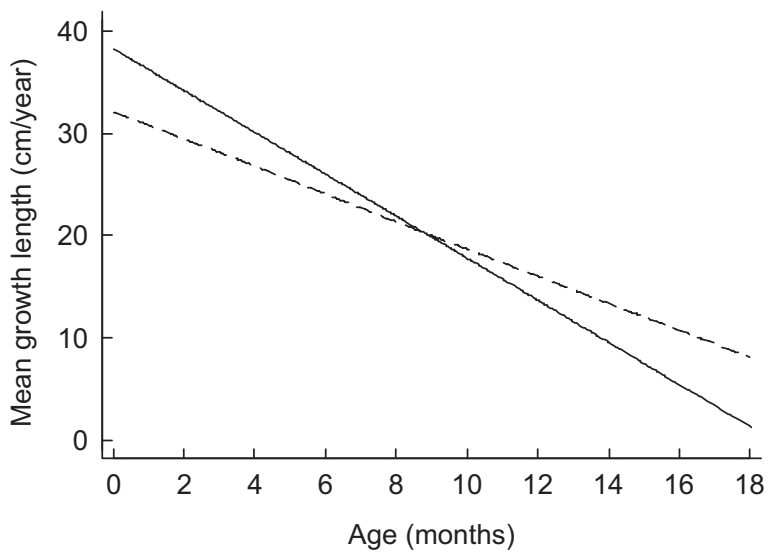

Fig. 2 Growth rate, expressed in terms of change in length (cm/year), of the uninfected (-) and HIV-infected (- - -) groups within a cohort of infants born between 1995 and 2004 in Belo Horizonte, Minas Gerais, Brazil

With respect to European children, it has previously been reported that while uninfected and HIV-infected infants did not present significant weight differences at the time of birth, the latter group was on average $460 \mathrm{~g}$ lighter at 3 months of age ${ }^{(3)}$. Moreover, the authors observed that the difference in weight between the two groups increased with age, confirming similar findings reported by Berhane et al. ${ }^{(18)}$. In this latter study, 6-month-old HIV-infected children from a Ugandan cohort were shown to present significantly lower weight and length than the uninfected group. The authors suggested that reduced weight and length resulted from general debilitation produced by frequent opportunistic infections and the consequent effects on food ingestion, absorption of nutrients and energy expenditure. Furthermore, there is evidence in the literature that viral replication itself is associated with growth impairment ${ }^{(19,20)}$.

Comparing the Belo Horizonte and the European cohorts $^{(3)}$, it was possible to establish that HIV-infected and uninfected children of all age groups belonging to the former presented lower growth rates by weight than the latter. Within the uninfected group, the growth rate by length in children up to 11 months old included in the Belo Horizonte cohort was larger than in the European cohort, while in children of other age groups the growth rate was smaller. HIV-infected children up to 5 months old presented no differences concerning growth rate by length. Children of the Belo Horizonte cohort within the age range of 6-12 months presented a larger growth rate by length than those of the European study ${ }^{(3)}$. It should be emphasised, however, that the comparison between children aged between 12 and 24 months must be viewed with caution, since the follow-up period for the Belo Horizonte cohort was only 18 months.

In both studies, there were HIV-infected children who had been treated with ART, as well as those who had not been so treated. A comparison of the growth rates of 
these two groups indicates that the main problem associated with the HIV-infected and uninfected children of the Belo Horizonte cohort related to factors associated with gain of body weight. The results of the present study, when compared with the European cohort, suggest that HIV-infected children of the Belo Horizonte cohort were more affected in terms of weight than in terms of length.

Since there were two types of individuals, i.e. ART treated and non-treated, within the HIV-infected group, various stages of the disease along with differential treatments could be found. Therefore, the diminished nutritional status observed amongst the HIV-infected children may be a consequence of the dissimilar progress of the disease in treated and non-treated children, or it could be associated with factors that were not evaluated in the present study (e.g. diminished ingestion of food, associated infections, secondary effects of medication, resistance to ART and adherence to treatment).

Since the children of the Belo Horizonte cohort were regularly followed up in a reputable specialised HIV centre, and many were born of mothers submitted to prenatal examination in the same institution, it is unlikely that these results are a consequence of a selection bias. The absence of food ingestion, socio-economic and clinical information in the files of the children of the Belo Horizonte cohort implied that it was not possible to establish whether the diminished growth observed among HIV-infected children was caused by the reduced ingestion of food and nutrients, side-effects of medication, associated infections or the evolution of the disease.

The anthropometric indicators derived from the present study suggest the need to identify HIV-infected children as early as possible and to adopt intervention strategies related to nutritional input.

\section{Acknowledgements}

Conflicts of interest: There is no conflict of interest that the authors should disclose.

Author contribution: M.A.F., epidemiologist, performed the statistical analysis. M.C., epidemiologist, helped with study design and interpretation of the results. E.A.C., statistician, helped with statistics. C.M.F.A., epidemiologist, helped with the interpretation of the results. J.A.P., paediatrician, group coordinator, was responsible for the selection of the cohort of children born from HIVinfected mothers.

Acknowledgements: The presented work was supported by Conselho Nacional de Desenvolvimento Cientifico e Tecnológico (CNPq), Fundação de Amparo à Pesquisas do Estado de Minas Gerais (FAPEMIG), and Coordenação de Aperfeiçoamento de Pessoal de Nível Superior (CAPES). 


\section{References}

1. UNAIDS (2004) Report on the Global AIDS Epidemic. Fourth Global Report. http://www.unaids.org/bangkok 2004/GAR2004_html/GAR2004_00_en.htm (accessed June 2006).

2. Ministério da Saúde (2005) Boletim Epidemiológico - AIDS e DST, Ano II, No. 1. Brasília: Ministério da Saúde.

3. Newell ML, Borja MC \& Peckham C (2003) Height, weight, and growth in children born to mothers with HIV-1 infection in Europe. Pediatrics 111, e52-e60.

4. Moye J, Rich KC, Kalish LA, Sheon AR, Diaz C, Cooper ER, Pitt J \& Handelsman E, for the Women and Infants Transmission Study Group (1996) Natural history of somatic growth in infants born to women infected by human immunodeficiency virus. J Pediatr 128, 58-67.

5. Carey VJ, Yong FH, Frenkel LM \& McKinney RM (2004) Growth velocity assessment in paediatric AIDS: smoothing, penalized quantile regression and the definition of growth failure. Stat Med 23, 509-526.

6. Chearskull S, Chotpitayasunondh $\mathrm{T}$, Simonds RJ et al. (2002) Survival, disease manifestations, and early predictors of disease progression among children with perinatal human immunodeficiency virus infection in Thailand. Pediatrics 10, e25; http://www.pediatrics.org/cgi/content/ full/110/2/e25

7. Verweel G, van Rossum AMC, Hartwig NG, Wolfs TFW, Scherpbier HJ \& Groot R (2002) Treatment with highly active antiretroviral therapy in human immunodeficiency virus type 1-infected children is associated with a sustained effect on growth. Pediatrics 109, e25; http://www. pediatrics.org/cgi/content/full/109/2/e25

8. Ministério da Saúde (2004) Guia de Tratamento Clínico da Infecção pelo HIV em Crianças. Série Manuais no. 18. Brasilia: Ministério da Saúde.

9. Centers for Disease Control and Prevention (1994) Revised classification system for human immunodeficiency virus infection in children less than 13 years of age. MMWR Morb Mortal Wkly Rep 43, 1-13.

10. World Health Organization (1995) Physical Status: the Use and Interpretation of Anthropometry. WHO Technical Report Series no. 854. Geneva: WHO.

11. MathSoft Inc. (1999) S-Plus 2000. Professional edition release 1. Seattle, WA: MathSoft Inc.

12. Stata Corp (1999) Stata statistics software: release 6.0. College Station, TX: Stata Corp.

13. Armitage P, Berry G \& Matthews JNS (2002) Statistical Methods in Medical Research, 4th ed. London: Blackwell Science.

14. Pinheiro JC \& Bates DM (2000) Mixed-effects Models in S and S-Plus. New York: Springer-Verlag.

15. Diggle PJ, Liang K-Y \& Zeger SL (1994) Analysis of Longitudinal Data. Oxford: Clarendon Press.

16. Guillen S, Ramos JT, Resino R, Bellón JM \& Muñoz MA (2007) Impact on weight and height with the use of HAART in HIV-infected children. Pediatr Infect Dis J 26, 334-338.

17. Nachman SA, Lindsey JC, Moye J, Stanley KE, Johnson GM, Krogstad PA, Wiznia AA \& Pediatric AIDS Clinical Trials Group 377 Study Team (2005) Growth of human immunodeficiency virus-infected children receiving highly active antiretroviral therapy. Pediatr Infect Dis $J \mathbf{2 4}$, 352-357.

18. Berhane R, Bagenda D, Marum L, Aceng E, Ndugwa C, Bosch RJ \& Olness K (1997) Growth failure as a prognostic indicator of mortality in pediatric HIV infection. Pediatrics 100, e7.

19. Henderson RA, Hutton N, Derusso P \& Caballero B (1997) Viral load is associated with nutritional status in HIV-infected children. Nutrition 13, 269.

20. Arpadi SM, Cuff PA, Kotler DP, Wang J, Bamji M, Lange M, Pierson RN \& Matthews DE (2000) Growth velocity, fat-free mass and energy intake are inversely related to viral load in HIV-infected children. J Nutr 130, 2498-2502. 